\title{
Clinical profile of recurrent vesicular palmoplantar dermatitis in children and adolescents
}

This article was published in the following Dove Press journal: Clinical, Cosmetic and Investigational Dermatology

\author{
Hugo Guimarães \\ Scotelaro-Alves' \\ Nurimar Conceição \\ Fernandes ${ }^{2}$ \\ Marcia Ramos-e-Silva ${ }^{3}$ \\ 'Sector of Dermatology, Post- \\ Graduation Program in Medical \\ Clinics, School of Medicine, Federal \\ University of Rio de Janeiro, Rio de \\ Janeiro, Brazil; ${ }^{2}$ Sector of Dermatology \\ and Institute of Pediatrics, School of \\ Medicine, Federal University of Rio de \\ Janeiro, Rio de Janeiro, Brazil; ${ }^{3}$ Sector \\ of Dermatology, School of Medicine, \\ Federal University of Rio de Janeiro, \\ Rio de Janeiro, Brazil
}

Correspondence: Hugo Guimarães Scotelaro-Alves

Sector of Dermatology, Post-Graduation Program in Medical Clinics, School of Medicine, Federal University of Rio de Janeiro, Av Afonso Arinos de Melo Franco 285/905, 2263 I-455 Rio de Janeiro, Brazil

Tel +55 2l 992332442

Email dermatohugo@gmail.com
Background: Previously known as dyshidrosis, recurrent vesicular palmoplantar dermatitis (RVPD) is presented as severe eruption of nonerythematous, symmetrical vesicles or bullae located along the lateral sides of fingers, on the palmar or plantar areas, and developing into a chronic and recurrent condition. Although very frequently observed on the hands, there are no specific studies about such eczema in children and adolescents.

Objectives: To report on the RVPD clinical profile in children and adolescents, and monitor the association of RVPD with seasonal variations, hyperhidrosis, atopy and nickel sulfate.

Materials and methods: Eighteen patients affected by RVPD were submitted to clinical and laboratory assessment through anamnesis, physical exam, mycological exam, patch test, complete blood count and serum IgE levels.

Results: Seven patients (38.9\%) reported increased frequency of eruptions during the summer months; 12 patients $(66.7 \%)$ presented hyperhidrosis; in 14 cases $(77.8 \%)$, atopic factors were determined; reaction to nickel sulfate was positive in three patients $(16.7 \%)$.

Conclusion: RVPD onset may occur at an early age, developing into a chronic and recurrent infection. Hyperhidrosis and atopic status was established in the clinical profile and no connection between nickel sulfate and RVPD was observed.

Keywords: dyshidrosis, hand eczema, vesicular hand dermatitis, pompholyx, children, hyperhidrosis, nickel allergy, atopic dermatitis

\section{Introduction}

Recurrent vesicular palmoplantar dermatitis (RVPD) is the current and more accurate terminology for the condition that was previously referred to as dyshidrosis or "pompholyx." It is characterized by a severe eruption of symmetrical nonerythematous and self-limited vesicles or bullae located along the lateral sides of fingers, on palmar or plantar areas. It presents a chronic and recurrent pattern. Sweat duct obstruction was first described by Fox as the cause of sweat dysfunction; ${ }^{1}$ therefore, he coined the concept of dyshidrosis (difficulty in sweating). Several publications within the last 135 years have shown absence of relationship between dermatosis and sweat glands, hence the term dyshidrosis has become strictly incorrect. ${ }^{2-5}$

Until today the etiology of RVPD remains unknown. Because of its chronic and recurrent nature, detecting a causative agent has become necessary for more effective treatments. Several causes were suggested and investigated. The most significant possibilities were: fungal infections, autosensitization dermatitis (dermatophyte infections), hyperhidrosis, pharmaceutical drugs, contact allergy and atopy. ${ }^{6-10}$ 
Few reliable data regarding RVPD occurrence and prevalence is available. It may occur at any age and is more often observed in young adults (patients aged 20-40 years). By analyzing the data collected from the age group of children and adolescents, we found a lack of research in the field or very limited specific studies on the subject. ${ }^{11}$

The present study aims to describe the clinical profile of RVPD in children and adolescents, by specifically monitoring the association of this condition with months of the year, hyperhidrosis, atopic status and nickel sulfate sensitization.

\section{Materials and methods}

A cross-sectional observational study was performed at the Pediatric Dermatology Ambulatory of the Institute of Pediatrics clinic of the Federal University of Rio de Janeiro between the years 2004 and 2012.

Patients with a range of 5 months to 18 years and with a presumptive diagnosis of RVPD were included in the study.

The diagnosis was based on the following strict criteria to recognize RVPD: eruption of symmetrical vesicles or bullae of nonerythematous base, self-limited and recurrent; involution as the lesions undergo a fine desquamation in form of halo, exclusively located on palms, soles and inner sides of the fingers; and occasionally associated pruritus.

A complete blood count test was taken to evaluate eosinophilia. When the parasitological stool sample examination yielded at least three negative results, it was considered a minor criterion for atopic status. ${ }^{12}$

Mycological exams (10\% KOH) and fungal culture (on Sabouraud's medium with chloramphenicol and Mycosel agar) were performed at room temperature during a period of 30 days in order to exclude dermatophytosis and "id" reactions.

Patch test was performed with nickel sulfate and placed on the patients' back for 48 hours. Readings were taken at 48 and 120 hours. Test readings followed recommendations of the International Contact Dermatitis Research Group (questionable reaction, soft erythematous macule $(+/-)$; weak/nonvesicular reaction, with erythema, infiltration and papule (+); strong/nonvesicular reaction, with erythema, infiltration and papules $(++)$; reaction with confluent bullae $(+++)$; negative reaction $(-)$; irritant reaction [IR]). ${ }^{10}$

The reference values of total serum IgE levels according to age groups were as follows: newborn $(2.3 \mathrm{kUI} / \mathrm{mL}$ or below); from 1 month to 12 years of age $(20 \mathrm{kUI} / \mathrm{mL}$ or below); from 4 to 10 years of age (100 kUI/mL or below); age $>10$ years $(150 \mathrm{kUI} / \mathrm{mL}$ or below $)$.
Collected data included: phototype, sex, age, seasonal variations, hyperhidrosis, individual and family history of atopy, atopic stigmas and exposure to nickel sulfate (jewelry and dental braces).

Primary hyperhidrosis was characterized by visible and focal excessive sweating of unknown causes for at least 6 months, associated with at least two of the following aspects: bilateral and symmetrical form, more than one occurrence a week, onset before 25 years of age, positive family history, ceasing of sweating effect during sleep, absence of interference from outside temperature or triggered by stress.

Diagnosis of atopic status was defined by the following criteria: stigmas (eczematide-like, Dennies-Morgan fold, dark circles, atopic palms and diffuse xerosis), individual or family history (allergic rhinitis, asthmatic bronchitis and atopic eczema), elevated serum immunoglobulin E(IgE) level or eosinophilia. Three groups of atopic status have been established: 1) patients with atopic family history, 2) presence of stigmas and 3) patients with atopic family history and elevated $\operatorname{IgE}$ level.

\section{Ethics committee}

The University Hospital Research Ethics Committee approved the protocol (number 39386132), and as this research involves patients under the legal age for consent for research (3-16 years), the parents or legal guardians of all the participants provided written informed consent on their behalf.

\section{Statistical analysis}

The collected descriptive analysis presented data collected in a table expressing frequency $(\mathrm{N})$ and percentage $(\%)$ as categorical variables and mean, $\mathrm{SD}$, maximum and minimum for each variant. Results of inferential analysis were obtained by Fisher's exact test to examine relationship between categorical data. The criterion used for determining significance was the level of 5\%. Statistical analysis was performed by SAS ${ }^{\circledR}$ software, version 6.11 (SAS Institute, Inc., Cary, NC, USA).

\section{Results}

With regard to incidence in relation to gender, 12 patients were female (66.7\%) and 6 were male (33.3\%) (Table 1).

Age ranged from 3 to 16 years, with overall average of 8.4 years and average age of onset of 5.9 years. The earliest onset case was 5 months old and the latest was 15 years old (Table 1)

Considering the difficulties in accurately determining which months come under each season, patients and their 
legal representatives were asked to relate months of the year with RVPD onset or recurrence. The majority (61\%) were not able to relate to any period; however, $38.9 \%$ included at least January and February (summer months) in Brazil (Table 1).

Palmoplantar lesion was predominant, comprising $\sim 12$ cases $(66.7 \%)$. There was one single case of lesion restricted to the plantar area (Table 1).

Palmoplantar hyperhidrosis, evaluated by objective criteria, was observed in 12 cases $(66.7 \%)$. Fourteen cases were identified as atopic through application of diagnostic criteria (Table 2).

All 18 patients were patch tested and three reacted to nickel sulfate (Table 2). Only one out of three cases with positive reaction to nickel sulfate had been exposed to the substance (use of jewelry) (Table 2).

Table I General description of the trial sample: epidemiologic profile

\begin{tabular}{llll}
\hline Variable & Category & Number & Percentage \\
\hline Phototype & III & 10 & 55.6 \\
& IV & 5 & 27.8 \\
& V & 3 & 16.7 \\
Sex & Male & 6 & 33.3 \\
& Female & 12 & 66.7 \\
Age (years) & $8.4 \pm 4.5(3-16)$ & & \\
Onset age (years) & $5.9 \pm 4.3(0.4 \mathrm{I}-15)$ & & \\
Months of the & Unrelated & 11 & 61.1 \\
year & Jan-Feb & 1 & 5.6 \\
& Dec-Mar & 4 & 22.2 \\
& Dec-May & 1 & 5.6 \\
& June-Sep/Dec-Mar & 1 & 5.6 \\
Hands & Yes & 17 & 94.4 \\
& No & 1 & 5.6 \\
Feet & Yes & 13 & 72.2 \\
& No & 5 & 27.8
\end{tabular}

Notes: data are presented as mean \pm SD (lowest age and highest age).

Table 2 General description of the trial sample: clinical and laboratory

\begin{tabular}{llll}
\hline Variable & Category & Number & Percentage \\
\hline Hyperhidrosis & Present & 12 & 66.7 \\
Atopy & Absent & 6 & 33.3 \\
& Yes & 14 & 77.8 \\
Eosinophilia & No & 4 & 22.2 \\
& Yes & 4 & 22.2 \\
IgE & No & 14 & 77.8 \\
& Elevated & 9 & 50 \\
Nickel sulfate & Normal & 9 & 50 \\
& Positive & 3 & 16.7 \\
Exposure to nickel & Negative & 15 & 83.3 \\
& Yes & 4 & 22.2 \\
& No & 14 & 77.8 \\
\hline
\end{tabular}

\section{Discussion}

The number of patients $(\mathrm{n}=18)$ who completed the study was proved to be satisfactory, since the condition has a low incidence rate (prevalence $<4 \%$ per year at the ambulatory facility in which the study was performed).

Despite all the controversy, reviewing of the literature on RVPD has indicated that the illness can be easily diagnosed. If strictly applied, the criteria (severe eruption of recurrent vesicles or bullae [symmetrical and nonerythematous], which may involute toward a fine desquamation in the form of a halo, restricted to the palms, soles and sides of the fingers) may clearly distinguish its main differential diagnosis: contact dermatitis characterized by dyshidrosiform eruptions. ${ }^{10}$

Dyshidrosiform eruptions are not precisely defined, but are a syndrome common to dermatophytosis, pharmacodermia and allergic and primary irritant contact dermatitis. Clinical signs of dyshidrosiform eruptions are evident erythema on vesicles base, fissures, keratosis, manifestations beyond palmoplantar areas or progression course longer than 3 weeks (Figures 1-5). The bibliographical survey carried out for this study has revealed articles which assumed that dyshidrosis/RVPD and dyshidrosiform eruptions were signs of a single illness, leading to unreliable data and inaccurate results.

In relation to sex, 12 participants $(66.6 \%)$ of the trial sample were female; there is no clinical data available in literature about this dermatitis in children. At the same time, the female preponderance is most pronounced in the group of adults affected by RVPD., ${ }^{4,713}$ Valejo encountered a higher percentage $(76 \%)$ of female patients in this group, considering the same geographical area as our survey. ${ }^{4} \mathrm{We}$ can assume that maybe there is a natural prevalence of RVPD in females.

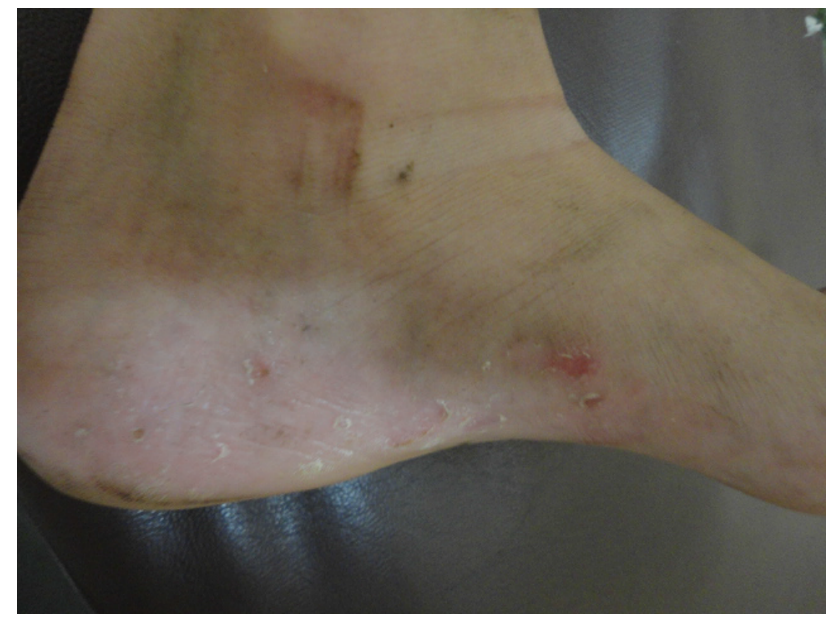

Figure I Vesicles, keratosis and fissures on the foot beyond the plantar region. 


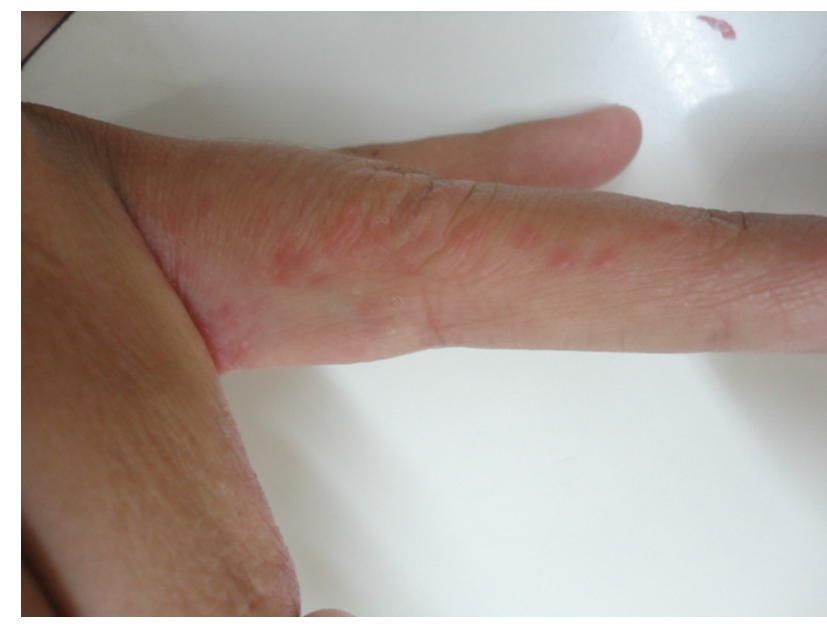

Figure 2 Vesicles on erythematous base on the side of a finger.

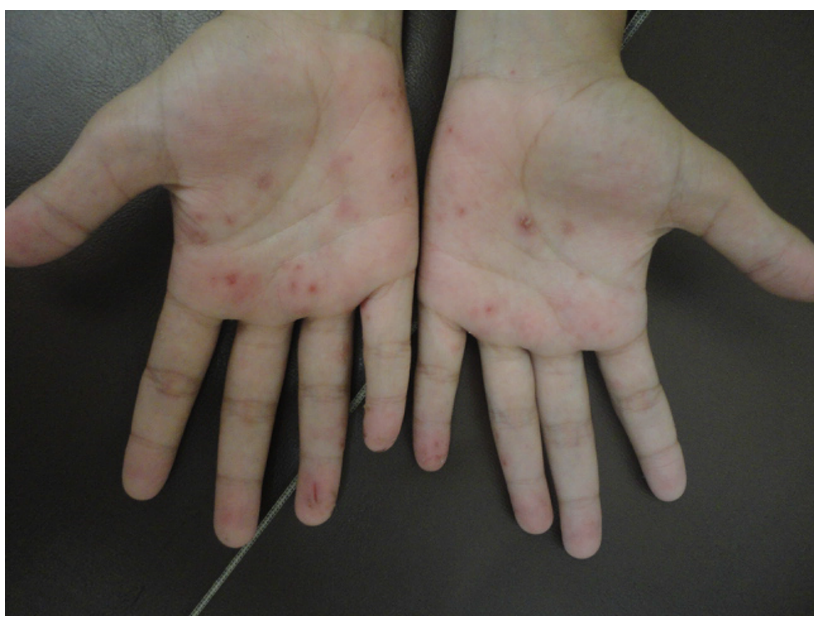

Figure 3 Vesicles and crusts on the palms and palmar surface of fingers.

Concerning the age of onset, $66.6 \%$ of cases were diagnosed before the age of 10 years. Of them, $44.4 \%$ were in preschool stage. Among all studies that assessed a large series of patients, there had been no evaluations under 10-year-old range., 4,6,7,13-17

In regards to RVPD progression, the chronic or recurrent aspect was observed from onset. It was not possible to crosscheck data given that our study was the only one among child/ adolescent age group, but is worth mentioning a publication on individuals ranging from 10 to 69 years, of whom $96 \%$ showed a progression course longer than 6 months. ${ }^{15}$

The occurrence or worsening of RVPD during the warmest months of the year was noticeable since the first reports ${ }^{1}$ and has always challenged researchers, especially, due to its correlation with primary hyperhidrosis. However, nowadays, it is known that such correlation is improper, since one of the criteria used to diagnose primary hyperhidrosis is the absence of interference from outside temperature.

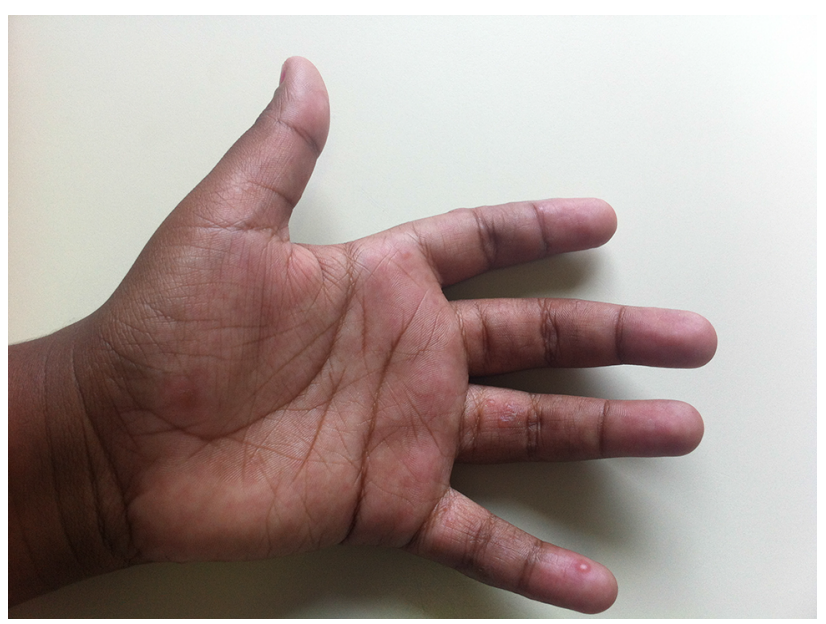

Figure 4 Vesicles on erythematous base on the side of fingers.

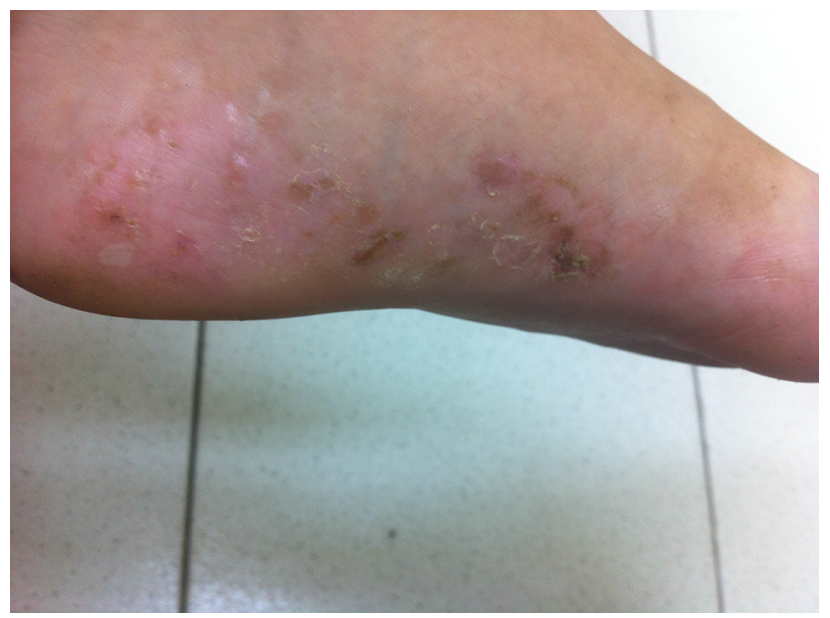

Figure 5 Vesicles and desquamation on the foot beyond the plantar region.

The majority of patients and legal representatives taking part in this study were not able to relate the manifestations to any period whatsoever; however, $38.9 \%$ related January and February (summer months) to clinical worsening. We should also take into consideration the climate conditions of the area to which the individual belongs (state of Rio de Janeiro) when contrasting with reviews of foreign literature concerning adults. ${ }^{6,13,15-17}$

As per topographical assessment, it was observed that hands and feet are simultaneously affected (66.6\%), followed by hands only (27.7\%) and just one patient reported feet only $(5.6 \%)$. Hutchinson proposed that occurrences would first affect the palmar area followed by the plantar. ${ }^{14}$ Approximately $46 \%$ of adult patients manifested lesions on palmoplantar areas at the same time. ${ }^{6}$

One of most relevant data of this study was attesting that none of the 18 cases presented a positive reaction to 
mycological or fungal culture exam on either palmar or plantar areas. This finding supports the fact that incidence of "tinea pedis" and "manuun" in children is rare. ${ }^{17,19}$

Twelve (66.6\%) patients were diagnosed with primary hyperhidrosis. Today, hyperhidrosis has been defined as a trigger/enhancer factor and no longer a causative agent of RVPD. During outpatient treatment, it was possible to observe palms that were often humid and cool to touch. Legal representatives themselves have linked the most intense period of hyperhidrosis to the triggering of RVPD episodes. Those periods of higher intensity were related to stress situations by both patients and their representatives (school tests, closeness to social events and family disruptions).

Hyperhidrosis seems to be a trigger/enhancer factor for RVPD among the age groups evaluated in this study. Three cases reported a higher intensity of hyperhidrosis symptoms and underwent treatment with $20 \%$ aluminum chloride solution for 5 consecutive days followed by three sessions per week, every other day. During treatment, they presented an increase of intercrisis period or even achieved effective control of RVPD. A hypothesis for the role of excessive sweating involved in the physiopathogenesis of RVPD is that a higher rate of sweating could activate the release of cytokines enhancing the inflammatory response, resulting in a local inflammatory reaction such as vesicles and bullae of RVPD. ${ }^{20}$

By comparing studies on hyperhidrosis with dyshidrosis among adults, Valejo reported this association in $28 \%$, Guillet in $40 \%$ and Lodi in $36.54 \%$ of cases. ${ }^{4,6,13}$ Although none of them was clear about what diagnostic criteria were used to define hyperhidrosis, we should bear in mind that the age group investigated in the present study exhibits a higher rate of transpiration naturally in comparison to adults.

Since this study aimed to analyze the relationship between atopy and RVPD, 14 patients were considered atopic (77.8\%). It is important to report that none of the 18 patients presented signs of active atopic eczema during the study; therefore, we assume that RVPD is not a manifestation of atopic eczema. We believe that there is a predisposition in atopic status individuals toward RVPD. Hence, we speculate that atopic patients are more prone to trigger RVPD due to certain aspects of their naturally enhanced inflammatory response.

The major controversy associated with RVPD is probably the connection with either exogenous (topical) or endogenous allergens. By definition, all patients with RVPD and positive patch test reaction to any substance with plausible evidence of causality have their accurate diagnosis of contact dermatitis characterized by dyshidrosiform eruptions.

It is interesting to imagine the existing causality between children and adolescents and exogenous agents causing contact dermatitis in palmoplantar areas, since adults exposed to a greater amount of products and allergens rarely develop contact dermatitis on the palmar and plantar areas at the same time. ${ }^{21,22}$ Toledo et al, in a study among children, found allergic contact dermatitis more frequently than atopic dermatitis and recommended patch testing in all children with chronic hand eczema, as already used in adults. ${ }^{23}$

In regards to the potential occurrence of dyshidrosiform eruptions by endogenous exposure, literature is not consensual. Furthermore, the studies with most favorable results presented adult patients exposed to concentration levels of nickel sulfate or cobalt chloride higher than normal diet levels. ${ }^{5,24,25}$ During the project development of this study, it was established that even if the hypothesis of endogenous allergens - behaving as trigger factors - is proven correct, a restrictive diet treatment for children and adolescents (most of them with low income) would not be justifiable.

All 18 patients were submitted to patch testing. Nickel sulfate is the most common triggering agent of RVPD and dyshidrosiform eruptions and is found in metal alloys (bracelets, rings, watches, dental braces).

Only one of the three patients with positive reaction to nickel sulfate was actually using jewelry (bracelet). According to the information given by the patient's mother, there was never a lesion on the part of skin in contact with the bracelet.

\section{Conclusion}

These results reinforce the impression that allergens do not influence the occurrence and triggering of RVPD in children and adolescents.

From our data, it is possible to suggest a clinical profile of children and adolescents with RVPD: female preponderance; age at onset as early as preschool years; development into a chronic infection; the warmest months of the year may contribute to enhance RVPD symptoms in some patients; palmoplantar areas are usually affected simultaneously, with a rare presentation of lesions involving plantar areas only; hyperhidrosis seems to be a relevant causing and worsening agent; atopic individuals are prone to develop RVPD; and nickel sulfate is not likely accounted for RVPD onset.

\section{Disclosure}

The authors report no conflicts of interest in this work. 


\section{References}

1. Fox T. Clinical lecture on dysidrosis: an undescribed eruption. Br Med J. 1873;2(665):365-366.

2. Robinson AR. Pompholyx: A Manual of Dermatology. New York: D. Appleton and Company, 1885: 246-255.

3. Shelley WB. Dyshidrosis (pompholyx). AMA Arch Dermatol. 1953; 68(3):314-319.

4. Valejo F. Dyshidrosis: Correlation with Atopy and with Sweat Gland. [master's thesis]. Rio de Janeiro, Brazil: UFRJ; 1993.

5. Storrs FJ. Acute and recurrent vesicular hand dermatitis not pompholyx or dyshidrosis. Arch Dermatol. 2007;143(12):1578-1580.

6. Lodi A, Betti R, Chiarelli G, Urbani CE, Crosti C. Epidemiological, clinical and allergological observations on pompholyx. Contact Dermatitis. 1992;26(1):17-21.

7. Minelli L. Dyshidrosis: prospective study of 600 cases. An Bras Dermatol. 1992;67(2):73-76..,.

8. Magina S, Barros MA, Ferreira JA, Mesquita-Guimarães J. Atopy, nickel sensitivity, occupation, and clinical patterns in different types of hand dermatitis. Am J Contact Dermat. 2003;14(2):63-68.

9. Bryld LE, Agner T, Menné T. Relation between vesicular eruptions on the hands and tinea pedis, atopic dermatitis and nickel allergy. Acta Derm Venerol. 2003;83(3):186-188.

10. Agner T, Aalto-Korte K, Andersen KE, et al; European Environmental and Contact Dermatitis Research Group. Classification of hand eczema. J Eur Acad Dermatol Venereol. 2015;29(12):2417-2422.

11. Ortiz-Salvador JM, Esteve-Martínez A, García-Rabasco A, SubiabreFerrer D, Martínez-Leboráns L, Zaragoza-Ninet V. Dermatitis of the foot: epidemiologic and clinical features in 389 children. Pediatr Dermatol. Epub 2017 Jul 21.

12. Hanifin JM, Rajka G. Diagnostic features of atopic eczema. Acta Derm Venerol Suppl (Stockh). 1980;60(92):44-47.
13. Guillet MH, Wierzbicka E, Guillet S, Dagregorio G, Guillet G. A 3 -year causative study of pompholyx in 120 patients. Arch Dermatol. 2007;143(12):1504-1508.

14. Hutchinson J. Cheiro-pompholyx. Br Med J. 1878;1(889):54.

15. Agrup G. Hand eczema and other hand dermatoses in South Sweden. Acta Derm Venerol (Stockh). 1969;49(Suppl 61):5-91.

16. Meding B. Epidemiology of hand eczema in an industrial city. Acta Derm Venerol. 1990;153:1-43.

17. Lofgren SM, Warshaw EM. Dyshidrosis: epidemiology, clinical characteristics, and therapy. Dermatitis. 2006;17(4):165-181.

18. Lehucher-Michel MP, Koeppel MC, Lanteaume A, Sayag J. Dyshidrotic eczema and occupation: a descriptive study. Contact Dermatitis. 2000;43(4):200-205.

19. Weston JA, Hawkins K, Weston WL. Foot dermatitis in children. Pediatrics. 1983;72(6):824-827.

20. Lee WJ, Lee DW, Kim CH, et al. Pomphollyx with bile-coloured vesicles in a patient with jaundice: are sweat ducts involved in the development of pompholyx? J Eur Acad Dermatol Venerol. 2010;24(2): 235-236.

21. Jain VK, Aggarwal K, Passi S, Gupta S. Role of contact allergens in pompholyx. J Dermatol. 2004;31(3):188-193.

22. Nishizawa A. Dyshidrotic eczema and its relationship to metal allergy. Curr Probl Dermatol. 2016;51:80-85.

23. Toledo F, García-Bravo B, Fernández-Redondo V, et al. Patch testing in children with hand eczema: a 5-year multicentre study in Spain. Contact Dermatitis. 2011;65(4):213-219.

24. Veien NK, Hattel T, Laurberg G. Low nickel diet: an open, prospective trial. J Am Acad Dermatol. 1993;29(6):1002-1007.

25. Fowler JF Jr, Storrs FJ. Nickel allergy and dyshidrotic eczema: are they related? Am J Contact Dermat. 2001;12(2):119-121.
Clinical, Cosmetic and Investigational Dermatology

\section{Publish your work in this journal}

Clinical, Cosmetic and Investigational Dermatology is an international, peer-reviewed, open access, online journal that focuses on the latest clinical and experimental research in all aspects of skin disease and cosmetic interventions. This journal is included on PubMed. The manuscript management system is completely online

\section{Dovepress}

and includes a very quick and fair peer-review system, which is all easy to use. Visit http://www.dovepress.com/testimonials.php to read real quotes from published authors 